Published in "Methods and Applications of Analysis", 2019, vol. 26, no 2, pp. 113-132, which should be cited to refer to this work.

DOI : $0.4310 / \mathrm{MAA} .2019 . \mathrm{v} 26 . \mathrm{n} 2 . \mathrm{a} 2$

\title{
A LEAST-SQUARES/RELAXATION METHOD FOR THE NUMERICAL SOLUTION OF A 2D PUCCI'S EQUATION
}

\author{
Alexandre Caboussat \\ Dedicated to Professor Roland Glowinski on the occasion of his 80th birthday
}

\begin{abstract}
The numerical solution of the Dirichlet problem for an elliptic Pucci's equation in two dimensions of space is addressed by using a least-squares approach. The algorithm relies on an iterative relaxation method that decouples a variational linear elliptic PDE problem from the local nonlinearities. The approximation method relies on mixed low order finite element methods.

The least-squares framework allows to revisit and extend the approach and the results presented in (Caffarelli, Glowinski, 2008) to more general cases. Numerical results show the convergence of the iterative sequence to the exact solution, when such a solution exists. The robustness of the approach is highlighted, when dealing with various types of meshes, domains with curved boundaries, non-convex domains, or nonsmooth solutions.
\end{abstract}

\section{Introduction}

Fully nonlinear elliptic equations have drawn a lot of attention lately, in the wake of the Monge-Ampère equation [7, 24]. Theoretical investigations of fully nonlinear equations $[2,17,28]$, are now related to numerous models and applications, for instance in finance [32], in seismic wave propagation [11], in geostrophic flows [14], in differential geometry [16], or in mechanics and physics. From the computational viewpoint, many approaches have been designed, both with finite differences $[2,11]$, or with finite element methods $[6,15,21]$.

Among those equations, the elliptic Pucci's equation, despite not being the most well-known fully nonlinear equation, has been addressed theoretically, e.g., in $[13,28]$. Special solutions have been obtained (see, e.g., [27]), and the eigenvalues (see, e.g., $[4,30]$ ), and bifurcation phenomena (see, e.g., [12]) have been studied. Meanwhile, a few dedicated computational methods have been presented in, e.g., $[8,9,10,26,31]$.

In this work, we revisit the approach from (Caffarelli, Glowinski, 2008) [8] for the solution of the Dirichlet problem for a two-dimensional elliptic Pucci's equation and extend their results. Namely, we use a least-squares approach and a relaxation algorithm, instead of considering splitting methods for the associated flow problem. This allows in particular to reduce the number of numerical parameters. Moreover, we incorporate structured and non-structured meshes, and arbitrary domains, which may be non-convex or with curved boundaries.

Key words and phrases. Pucci's equation, Least-squares method, Nonlinear constrained minimization, Newton method. Mixed finite elements.

Received August 13, 2019 
More precisely, we consider here a bounded, smooth, two-dimensional domain $\Omega \subset \mathbb{R}^{2}$. For boundary data $g: \partial \Omega \rightarrow \mathbb{R}$ sufficiently regular, the Dirichlet problem for the $2 \mathrm{D}$ Pucci's equation consists in looking for a function $\psi: \Omega \rightarrow \mathbb{R}$ satisfying

$$
\begin{array}{ll}
\alpha \lambda^{+}+\lambda^{-}=0 & \text { in } \Omega \subset \mathbb{R}^{2}, \\
\psi=g & \text { on } \partial \Omega,
\end{array}
$$

where $\lambda^{+}, \lambda^{-}$are the largest and smallest eigenvalues of the Hessian $\mathbf{D}^{2} \psi$ of the unknown function $\psi$, and $\alpha>1$ is a given parameter. Note that, in three space dimensions, the Pucci's equation consists in looking for a function $\psi: \Omega \rightarrow \mathbb{R}$ satisfying

$$
\begin{array}{ll}
\alpha \sum \lambda^{+}+\sum \lambda^{-}=0 & \text { in } \Omega \subset \mathbb{R}^{3} \\
\psi=g & \text { on } \partial \Omega
\end{array}
$$

where $\lambda^{+}$and $\lambda^{-}$are the positive and negative eigenvalues of the Hessian $\mathbf{D}^{2} \psi$ respectively. Fully nonlinear equations do not always admit classical solutions, see, e.g., [7], and a concept of weak solutions has been introduced, based on Aleksandrov solutions [1], or viscosity solutions [25, 28]. The least-squares approach we introduce here also allows to define weak solutions in some sense when classical solutions do not exist [6]. However, they do not necessarily correspond to viscosity solutions in the classical sense.

The chosen least-squares formulation introduced here consists in minimizing the $L^{2}$-distance between the Hessian $\mathbf{D}^{2} \psi$ and an auxiliary variable $\mathbf{p}$ in $L^{2 \times 2}(\Omega)$, where $\psi$ satisfies the boundary conditions of the problem (but not the Pucci's equation) and $\mathbf{p}$ satisfies point-wise, algebraically, the Pucci's equation (without considering any boundary conditions). Using a relaxation algorithm to minimize such a distance allows to decouple the solution method into a sequence of linear variational problems (solved with conforming finite element techniques), and local nonlinear algebraic minimization problems (solved with dedicated solvers). Linear variational problems are solved with a conjugate gradient algorithm [19], coupled with a mixed finite element method based on $\mathbb{P}_{1}$ finite elements, Local nonlinear problems (namely constrained minimization problems locally at each point of the domain $\Omega$ ) are solved with Newton-type techniques, see e.g. [33].

The structure of this article is as follows. In Section 2, we describe the model problem and the proposed methodology, while the relaxation algorithm is described in Section 3. Sections 4 and 5 detail the algebraic and differential solvers respectively. The mixed finite element discretization is proposed in Section 6, and numerical experiments are treated in Section 7 for validation purposes and sensitivity analysis.

\section{Pucci's Equation and Least-Squares Approach}

Let $\Omega$ be a bounded convex domain of $\mathbb{R}^{2}$, with boundary $\partial \Omega$ and $\left.\alpha \in\right] 1, \infty[$ a given parameter. Let us consider boundary data $g: \partial \Omega \rightarrow \mathbb{R}$ that is sufficiently regular, The Dirichlet problem for the Pucci's equation reads as follows: find $\psi \in H^{2}(\Omega)$ satisfying

$$
\begin{cases}\alpha \lambda^{+}+\lambda^{-}=0 & \text { in } \Omega \\ \psi=g & \text { on } \partial \Omega\end{cases}
$$


where $\lambda^{+}, \lambda^{-}$are respectively the largest and smallest eigenvalues of the Hessian $\mathbf{D}^{2} \psi$ of the unknown function $\psi$ (defined by $\mathbf{D}^{2} \psi=\left(D_{i j}^{2} \psi\right)_{1 \leq i, j \leq 2}$, where $\left.D_{i j}^{2} \psi=\frac{\partial^{2} \psi}{\partial x_{i} \partial x_{j}}\right)$. Note that, if $\alpha \rightarrow 1$, (1) reduces to the Poisson-Dirichlet problem $\Delta \psi=0$ in $\Omega$, with boundary conditions $\psi=g$ on $\partial \Omega$.

Following [8], a calculation shows that (1) is indeed a fully nonlinear equation, as it is equivalent to: find $\psi \in H^{2}(\Omega)$ satisfying

$$
\begin{cases}\alpha|\Delta \psi|^{2}+(\alpha-1)^{2} \operatorname{det} \mathbf{D}^{2} \psi=0 & \text { in } \Omega, \\ \Delta \psi \leq 0 & \text { in } \Omega, \\ \psi=g & \text { on } \partial \Omega\end{cases}
$$

This relation shows that the Pucci equation combines the Laplace operator $\Delta$ and the Monge-Ampère operator $\operatorname{det} \mathbf{D}^{2}$. Moreover, this expression shows that the Pucci's equation is actually equivalent to

$$
\begin{cases}\mathbf{p}=\mathbf{D}^{2} \psi & \\ \alpha\left(p_{11}+p_{22}\right)^{2}+(\alpha-1)^{2}\left(p_{11} p_{22}-p_{12}^{2}\right)=0 & \text { in } \Omega \\ p_{11}+p_{22} \leq 0 & \text { in } \Omega \\ \psi=g & \text { on } \partial \Omega\end{cases}
$$

with $\mathbf{p}=\mathbf{p}^{T}$ and $p_{i j}=\frac{\partial^{2} \psi}{\partial x_{i} \partial x_{j}}, i, j=1,2$.

Let us consider in the sequel $g \in H^{3 / 2}(\partial \Omega)$. We use the Fröbenius norm and product respectively defined by $|\mathbf{T}|=(\mathbf{T}: \mathbf{T})^{1 / 2}, \mathbf{S}: \mathbf{T}=\sum_{i, j=1}^{2} s_{i j} t_{i j}$ for each $\mathbf{S}=\left(s_{i j}\right), \mathbf{T}=\left(t_{i j}\right) \in \mathbb{R}^{2 \times 2}$. A numerical method of the nonlinear leastsquares type is advocated for the solution of (2), similar to the methodology presented in [6]. More precisely we want to solve the equivalent problem: find $(\psi, \mathbf{p}) \in V_{g} \times \mathbf{Q}_{P}$ satisfying

$$
J(\psi, \mathbf{p}) \leq J(\varphi, \mathbf{q}), \quad \forall(\varphi, \mathbf{q}) \in V_{g} \times \mathbf{Q}_{P},
$$

where $J(\varphi, \mathbf{q})=\frac{1}{2} \int_{\Omega}\left|\mathbf{D}^{2} \varphi-\mathbf{q}\right|^{2} d \mathbf{x}$. The functional spaces in (3) are respectively defined by:

$$
\begin{aligned}
V_{g} & =\left\{\varphi \in H^{2}(\Omega), \varphi=g \text { on } \partial \Omega\right\} \\
\mathbf{Q} & =\left\{\mathbf{q} \in L^{2}(\Omega)^{2 \times 2}, \mathbf{q}=\mathbf{q}^{T}\right\} \\
\mathbf{Q}_{P} & =\left\{\mathbf{q} \in \mathbf{Q}, \alpha\left(q_{11}+q_{22}\right)^{2}+(\alpha-1)^{2}\left(q_{11} q_{22}-q_{12}^{2}\right)=0, q_{11}+q_{22} \leq 0\right\} .
\end{aligned}
$$

The space $\mathbf{Q}$ is a Hilbert space for the scalar product $\left(\mathbf{q}, \mathbf{q}^{\prime}\right) \rightarrow \int_{\Omega} \mathbf{q}: \mathbf{q}^{\prime} d \mathbf{x}$, and the associated norm $\|\mathbf{q}\|=(\mathbf{q}, \mathbf{q})^{1 / 2}$. Note that the existence and uniqueness of a solution to the least-squares problem (3) is, to the best of our knowledge, still an open research topic and has not been proved yet. However, we can see that a solution to (3) that corresponds to a vanishing objective function $J(\cdot, \cdot)$, is also a solution to the original problem (1), by construction of the functional spaces $\mathbf{Q}_{P}$ and $V_{g}$. When the objective function $J(\cdot, \cdot)$ does not vanish, the solution to (3) is the best one in a least-squares sense. Numerical results will show that, for all numerical experiments considered, the proposed algorithm finds the exact solution each time that such an exact solution exists. 


\section{Relaxation Algorithm}

Following [8], we propose a relaxation algorithm in order to solve (3), which allows to decouple nonlinearities and variational problems. We initialize the algorithm with the solution of

$$
-\Delta \psi^{0}=0, \quad \text { in } \Omega, \quad \psi^{0}=g, \quad \text { on } \partial \Omega .
$$

This solution corresponds to the limit case $\alpha=1$ in (1), and therefore, the smaller the value of $\alpha$, the better the initial guess of the algorithm. Then, for $n \geq 0$, assuming that $\psi^{n}$ is known, we compute $\mathbf{p}^{n}, \psi^{n+1 / 2}$ and $\psi^{n+1}$ as follows:

$$
\begin{aligned}
\mathbf{p}^{n} & =\arg \min _{\mathbf{q} \in \mathbf{Q}_{P}} J\left(\psi^{n}, \mathbf{q}\right) ; \\
\psi^{n+1 / 2} & =\arg \min _{\varphi \in V_{g}} J\left(\varphi, \mathbf{p}^{n}\right) ; \\
\psi^{n+1} & =\psi^{n}+\omega\left(\psi^{n+1 / 2}-\psi^{n}\right) ;
\end{aligned}
$$

where $1 \leq \omega \leq \omega_{\max }<2$ is a relaxation parameter.

Similarly to so-called alternating direction implicit methods, this relaxation algorithm (4)-(6) alternates between the variables $\mathbf{p}^{n}$ and $\psi^{n+1}$, to update one unknown while keeping the other unknown fixed.

Such alternating direction schemes have been widely studied in the literature, in particular coupled with augmented Lagrangian algorithms [18, 23]. The relaxation step (6) is added with the main goal of accelerating the convergence speed of the iterative algorithm. A complete study of the optimal value of the relaxation parameter has not been undertaken, but numerical experiments have shown that the algorithm is robust when $\omega=1$. We refer to, e.g., [23] for further general results on the convergence of relaxation algorithms.

\section{Numerical Solution of the Local Optimization Problems}

An explicit formulation of (4) is given by

$$
\mathbf{p}^{n}=\arg \min _{\mathbf{q} \in \mathbf{Q}_{P}}\left[\frac{1}{2} \int_{\Omega}|\mathbf{q}|^{2} d \mathbf{x}-\int_{\Omega} \mathbf{D}^{2} \psi^{n}: \mathbf{q} d \mathbf{x}\right] .
$$

Since the objective function does not contain any derivatives of $\mathbf{q}$, the minimization problem (7) can be solved point-wise (in practice at the vertices of a finite element or finite difference mesh). A finite dimensional minimization problem is solved for a.e. $\mathbf{x}$ in $\Omega$ :

$$
\mathbf{p}^{n}(\mathbf{x})=\arg \min _{\mathbf{q} \in \mathbf{E}_{P}}\left[\frac{1}{2}|\mathbf{q}|^{2}-\mathbf{D}^{2} \psi^{n}(\mathbf{x}): \mathbf{q}\right]
$$

where

$$
\begin{aligned}
\mathbf{E}_{P}= & \left\{\mathbf{q} \in \mathbb{R}^{2 \times 2}, \mathbf{q}=\mathbf{q}^{T}, q_{11}+q_{22} \leq 0\right. \\
& \left.\alpha\left(q_{11}+q_{22}\right)^{2}+(\alpha-1)^{2}\left(q_{11} q_{22}-q_{12}^{2}\right)=0\right\} .
\end{aligned}
$$

This corresponds to a family of constrained quadratic optimization problems. By setting 


$$
\begin{aligned}
\left(z_{1}, z_{2}, z_{3}\right) & :=\left(q_{11}, q_{22}, q_{12}\right) \text { and } \\
\left(b_{1}, b_{2}, b_{3}\right) & :=\left(D^{2} \psi_{11}^{n}(\mathbf{x}), D^{2} \psi_{22}^{n}(\mathbf{x}), D^{2} \psi_{12}^{n}(\mathbf{x})\right)
\end{aligned}
$$

problem (8) is equivalent to solving

$$
\min _{\left(z_{1}, z_{2}, z_{3}\right) \in E_{3}}\left[\frac{1}{2}\left(z_{1}^{2}+z_{2}^{2}+z_{3}^{2}\right)-\left(b_{1} z_{1}+b_{2} z_{2}+b_{3} z_{3}\right)\right]
$$

over the set

$$
E_{3}=\left\{\left(z_{1}, z_{2}, z_{3}\right) \in \mathbb{R}^{3}, \alpha\left(z_{1}+z_{2}\right)^{2}+(\alpha-1)^{2}\left(z_{1} z_{2}-z_{3}^{2}\right)=0, z_{1}+z_{2} \leq 0\right\} .
$$

Defining $\gamma:=\frac{\alpha}{(\alpha-1)^{2}}$, and eliminating the variable $z_{3}$ through the equality constraint (with $\left.z_{3}^{2}=\gamma\left(z_{1}+z_{2}\right)^{2}+z_{1} z_{2}\right)$, (9) is equivalent to

$$
\begin{aligned}
\min _{\left(z_{1}, z_{2}\right) \in E_{2}} & {\left[\frac{1}{2}\left(z_{1}^{2}+z_{2}^{2}+\gamma\left(z_{1}+z_{2}\right)^{2}+z_{1} z_{2}\right)-b_{1} z_{1}-b_{2} z_{2}\right.} \\
& \left.-\left|b_{3}\right|\left(\gamma\left(z_{1}+z_{2}\right)^{2}+z_{1} z_{2}\right)^{1 / 2}\right]
\end{aligned}
$$

over the set

$$
E_{2}=\left\{\left(z_{1}, z_{2}\right) \in \mathbb{R}^{2}, \gamma\left(z_{1}+z_{2}\right)^{2}+z_{1} z_{2} \geq 0, z_{1}+z_{2} \leq 0\right\}
$$

together with $z_{3}=\operatorname{sign}\left(b_{3}\right)\left(\gamma\left(z_{1}+z_{2}\right)^{2}+z_{1} z_{2}\right)^{1 / 2}$. Setting $z_{1}=\rho \cos (\theta)$ and $z_{2}=\rho \sin (\theta)$, with $\rho \geq 0$ and $\theta \in[0,2 \pi)$, we verify that (10) is equivalent to

$$
\max _{\theta \in K_{\theta}} F(\theta)
$$

where

$$
F(\theta)=\left[\frac{\left(b_{1} \cos \theta+b_{2} \sin \theta+\left|b_{3}\right| \sqrt{\gamma+(0.5+\gamma) \sin (2 \theta)}\right.}{\sqrt{1+\gamma+(0.5+\gamma) \sin (2 \theta)}}\right],
$$

with $K_{\theta}=\left[\pi-\frac{1}{2} \phi_{c}, \frac{3}{2} \pi+\frac{1}{2} \phi_{c}\right]$ and $\phi_{c}=\sin ^{-1}[2 \gamma /(2 \gamma+1)]$. Let us denote by $\theta_{m}$ the solution to (11), which can be obtained by a safeguarded Newton method. The solution to (9) is thus given by $\left(z_{1}, z_{2}, z_{3}\right)=(0,0,0)$ if $F\left(\theta_{m}\right) \leq 0$, and, if $F\left(\theta_{m}\right)>0$, it is given by

$$
\left(\begin{array}{c}
z_{1} \\
z_{2} \\
z_{3}
\end{array}\right)=\left(\begin{array}{c}
\rho_{m} \cos \left(\theta_{m}\right) \\
\rho_{m} \sin \left(\theta_{m}\right) \\
\operatorname{sign}\left(b_{3}\right) \sqrt{\gamma \rho_{m}^{2}\left(\cos \left(\theta_{m}\right)+\sin \left(\theta_{m}\right)\right)^{2}+\rho_{m}^{2} \cos \left(\theta_{m}\right) \sin \left(\theta_{m}\right)}
\end{array}\right)
$$

where

$$
\rho_{m}=\frac{\left(b_{1} \cos \theta_{m}+b_{2} \sin \theta_{m}+\left|b_{3}\right| \sqrt{\gamma+(0.5+\gamma) \sin \left(2 \theta_{m}\right)}\right.}{1+\gamma+(0.5+\gamma) \sin \left(2 \theta_{m}\right)}
$$




\section{Numerical Solution of the Linear Variational Problems}

Written in variational form, the Euler-Lagrange equation of the minimization problem (5) reads as follows: find $\psi^{n+1 / 2} \in V_{g}$ such that

$$
\int_{\Omega} \mathbf{D}^{2} \psi^{n+1 / 2}: \mathbf{D}^{2} \varphi d \mathbf{x}=\int_{\Omega} \mathbf{p}^{n}: \mathbf{D}^{2} \varphi d \mathbf{x}, \quad \forall \varphi \in V_{0}
$$

where $V_{0}=H^{2}(\Omega) \cap H_{0}^{1}(\Omega)$. The, biharmonic-type, linear variational problem (12) is well-posed. It can be solved with a conjugate gradient algorithm operating in the spaces $V_{0}$ and $V_{g}$, both spaces being equipped with the scalar product defined by $(\psi, \varphi) \rightarrow \int_{\Omega} \Delta \psi \Delta \varphi d \mathbf{x}$, and the corresponding norm. A complete description of such conjugate gradient solution of linear variational problems in Hilbert spaces, such as (12), has been given in, e.g., [18, Chapter $3]$. Here, this conjugate gradient algorithm reads as follows:

\section{Initialization step:}

- Let $\psi^{n+1 / 2,0} \in V_{g}$ be given.

- Find $g^{0} \in V_{0}$ such that

$$
\int_{\Omega} \Delta g^{0} \Delta \varphi d \mathbf{x}=\int_{\Omega} \mathbf{D}^{2} \psi^{n+1 / 2,0}: \mathbf{D}^{2} \varphi d \mathbf{x}-\int_{\Omega} \mathbf{p}^{n}: \varphi d \mathbf{x}, \quad \forall \varphi \in V_{0} .
$$

- Set $w^{0}=g^{0}$.

Iteration step: For $k \geq 0, \psi^{n+1 / 2, k}, g^{k}$ and $w^{k}$ being known, the last two different from zero, we compute $\psi^{n+1 / 2, k+1}, g^{k+1}$ and, if necessary, $w^{k+1}$ as follows.

- Find $\bar{g}^{k} \in V_{0}$ satisfying

$$
\int_{\Omega} \Delta \bar{g}^{k} \Delta \varphi d \mathbf{x}=\int_{\Omega} \mathbf{D}^{2} w^{k}: \mathbf{D}^{2} \varphi d \mathbf{x}, \quad \forall \varphi \in V_{0}
$$

- Compute successively

$$
\begin{aligned}
\rho_{k} & =\left(\int_{\Omega}\left|\Delta g^{k}\right|^{2} d \mathbf{x}\right)\left(\int_{\Omega} \Delta \bar{g}^{k} \Delta w^{k} d \mathbf{x}\right)^{-1} ; \\
\psi^{n+1 / 2, k+1} & =\psi^{n+1 / 2, k}-\rho_{k} w^{k} ; \\
g^{k+1} & =g^{k}-\rho_{k} \bar{g}^{k} ; \\
\delta_{k} & =\left(\int_{\Omega}\left|\Delta g^{k+1}\right|^{2} d \mathbf{x}\right)\left(\int_{\Omega}\left|\Delta g^{0}\right|^{2} d \mathbf{x}\right)^{-1} .
\end{aligned}
$$

- If $\delta_{k}<\varepsilon$ (where $\varepsilon$ is a given tolerance), take $\psi^{n+1 / 2}=\psi^{n+1 / 2, k+1}$; otherwise, compute:

$$
\begin{aligned}
\gamma_{k} & =\left(\int_{\Omega}\left|\Delta g^{k+1}\right|^{2} d \mathbf{x}\right)\left(\int_{\Omega}\left|\Delta g^{k}\right|^{2} d \mathbf{x}\right)^{-1} ; \\
w^{k+1} & =g^{k+1}+\gamma_{k} w^{k} ;
\end{aligned}
$$

and repeat (go back to (14)).

Numerical experiments have shown that the conjugate gradient algorithm converges typically in less than 20 iterations for the range of mesh sizes considered (considering $\varepsilon=10^{-5}$ ). 
Concerning the solution of the bi-harmonic problems in (13) and (14), they are both of the following type:

$$
\text { Find } r \in V \text { such that } \int_{\Omega} \Delta r \Delta v d \mathbf{x}=\Lambda(v), \quad \forall v \in V,
$$

the functional $\Lambda(\cdot)$ being linear over $V$. Let us denote $-\Delta r$ by $\omega$. It follows that (16) is equivalent to the following system of two coupled, well-posed, Poisson-Dirichlet problems

$$
\begin{aligned}
& \omega \in V, \quad \int_{\Omega} \nabla \omega \cdot \nabla v d \mathbf{x}=\Lambda(v), \quad \forall v \in V, \\
& r \in V, \quad \int_{\Omega} \nabla r \cdot \nabla v d \mathbf{x}=\int_{\Omega} \omega v d \mathbf{x}, \quad \forall v \in V .
\end{aligned}
$$

This fundamentally allows to find solutions to the Pucci's equation by only solving local algebraic problems (point-wise), and elliptic Poisson problems.

\section{Mixed Finite Element Approximation}

Considering the highly variational flavor of the methodology discussed in the preceding sections, it makes sense to look for finite element based methods for the approximation of (1). We use a mixed finite element approximation (closely related to those discussed in, e.g., [19] for the solution of linear and nonlinear bi-harmonic problems) with piecewise linear $\mathbb{P}_{1}$ and globally continuous finite elements on a (structured or unstructured) discretization made out of triangles.

6.1. Finite Element Spaces. Let us define $\mathcal{T}_{h}$ a finite element discretization of $\Omega$ made out of triangles (see, e.g., [19, Appendix 1]). Let $\Sigma_{h}$ be the set of vertices of $\mathcal{T}_{h}, \Sigma_{0 h}=\left\{P \in \Sigma_{h}, P \notin \partial \Omega\right\}, N_{h}=\operatorname{Card}\left(\Sigma_{h}\right)$, and $N_{0 h}=$ $\operatorname{Card}\left(\Sigma_{0 h}\right)$. We suppose that $\Sigma_{0 h}=\left\{P_{j}\right\}_{j=1}^{N_{0 h}}$ and $\Sigma_{h}=\Sigma_{0 h} \cup\left\{P_{j}\right\}_{j=N_{0 h}+1}^{N_{h}}$.

From $\mathcal{T}_{h}$, we approximate the spaces $L^{2}(\Omega), H^{1}(\Omega)$ and $H^{2}(\Omega)$ by the finite dimensional space $V_{h}$ defined by:

$$
V_{h}=\left\{v \in C^{0}(\bar{\Omega}),\left.v\right|_{T} \in \mathbb{P}_{1}, \forall T \in \mathcal{T}_{h}\right\},
$$

with $\mathbb{P}_{1}$ the space of the two-variable polynomials of degree $\leq 1$. We also define $V_{0 h}$ as

$$
V_{0 h}=V_{h} \cap H_{0}^{1}(\Omega)=\left\{v \in V_{h}, v=0 \text { on } \partial \Omega\right\} .
$$

In the sequel, $V_{0 h}$ is the approximation space of $H_{0}^{1}(\Omega)$ and $H^{2}(\Omega) \cap H_{0}^{1}(\Omega)$.

The proposed method is based on mixed finite elements, as both variables $(\psi, \mathbf{p}) \in V_{g} \times \mathbf{Q}_{P}$ will be approximated in functional spaces relying both on piecewise linear finite elements. However the unknowns $\mathbf{p}$ and $\psi$ are decoupled, thanks to the Uzawa iterative algorithm. Therefore, practically, all the local nonlinear problems or global linear variational problems will be well-posed in the iterative algorithm. We refer to [8, 20,23] for further details; in particular $[3$, Chapter IV] treats inf-sup conditions for biharmonic problems.

6.2. Finite Element Approximation of the Pucci's Equation. When solving (12) with the conjugate gradient algorithm (13)-(15), one has to i) approximate numerical equivalents of the second order derivatives, e.g., $\mathbf{D}^{2} w^{k}$ and $\mathbf{D}^{2} u^{0}$, and ii) solve biharmonic problems such as (13) or (14). 
We would like to approximate numerical equivalents of the second order derivatives with piecewise linear finite elements, which has to be handled carefully. For a function $\varphi$ being given in $H^{2}(\Omega)$, it follows from Green's formula that, for $i, j=1,2$ :

$$
\int_{\Omega} \frac{\partial^{2} \varphi}{\partial x_{i} \partial x_{j}} v d \mathbf{x}=-\frac{1}{2} \int_{\Omega}\left[\frac{\partial \varphi}{\partial x_{i}} \frac{\partial v}{\partial x_{j}}+\frac{\partial \varphi}{\partial x_{j}} \frac{\partial v}{\partial x_{i}}\right] d \mathbf{x}, \forall v \in H_{0}^{1}(\Omega) .
$$

Consider now $\varphi \in V_{h}$. We define the approximations $D_{h i j}^{2} \varphi \in V_{0 h}$ of the second derivatives $\frac{\partial^{2} \varphi}{\partial x_{i} \partial x_{j}}$ by

$$
\int_{\Omega} D_{h i j}^{2} \varphi v d \mathbf{x}:=-\frac{1}{2} \int_{\Omega}\left[\frac{\partial \varphi}{\partial x_{i}} \frac{\partial v}{\partial x_{j}}+\frac{\partial \varphi}{\partial x_{j}} \frac{\partial v}{\partial x_{i}}\right] d \mathbf{x}, \forall v \in V_{0 h} .
$$

The functions $D_{h i j}^{2} \varphi$ are thus uniquely defined; in order to simplify the computation of the above discrete second order partial derivatives, we could use the trapezoidal rule to evaluate the integrals in the left hand side (mass lumping).

As emphasized in $[6,29]$, when using piecewise linear mixed finite elements, the a priori error estimates for the second derivatives of the solution $\psi$ are $\mathcal{O}(1)$ in the $L^{2}$-norm. Therefore, the convergence properties of the global algorithm strongly depends on the type of triangulation, and could be completely jeopardized in some situations. One way to regularize the approximation of the second derivatives $D_{h i j}^{2} \varphi$ and recover some convergence properties is to use a Tychonoff regularization [34]. Let us introduce a stabilization constant $C$ (to be calibrated in the numerical experiments), and replace the previous relationship by:

$$
\begin{aligned}
\int_{\Omega} D_{h i j}^{2} \varphi v d \mathbf{x}+C \sum_{K \in \mathcal{T}_{h}}|K| \int_{\Omega} \nabla D_{h i j}^{2} \varphi \cdot \nabla v d \mathbf{x} \\
=-\frac{1}{2} \int_{\Omega}\left[\frac{\partial \varphi}{\partial x_{i}} \frac{\partial v}{\partial x_{j}}+\frac{\partial \varphi}{\partial x_{j}} \frac{\partial v}{\partial x_{i}}\right] d \mathbf{x}, \forall v \in V_{0 h}
\end{aligned}
$$

Defining $\mathbf{D}_{h}^{2} \psi_{h}\left(P_{k}\right)=\left(D_{h i j}^{2} \psi_{h}\left(P_{k}\right)\right)_{i, j=1}^{2}$, and assuming that the boundary function $g$ is continuous over $\partial \Omega$, the affine space $V_{g}$ can be approximated by

$$
V_{g h}=\left\{\varphi \in V_{h}, \varphi(P)=g(P), \forall P \in \Sigma_{h} \cap \partial \Omega\right\}
$$

and (2) is approximated by its discrete version: find $\psi_{h} \in V_{g h}$ satisfying

$$
\begin{aligned}
& \alpha\left|D_{h 11}^{2} \psi_{h}\left(P_{k}\right)+D_{h 22}^{2} \psi_{h}\left(P_{k}\right)\right|^{2} \\
& \quad+(\alpha-1)^{2}\left(D_{h 11}^{2} \psi_{h}\left(P_{k}\right) D_{h 22}^{2} \psi_{h}\left(P_{k}\right)-D_{h 12}^{2} \psi_{h}\left(P_{k}\right)\right)=0
\end{aligned}
$$

with $D_{h 11}^{2} \psi_{h}\left(P_{k}\right)+D_{h 22}^{2} \psi_{h}\left(P_{k}\right) \leq 0$, for all $k=1, \ldots, N_{0 h}$.

6.3. Discrete Formulation of the Least-Squares Method. We define the discrete equivalents of $\mathbf{Q}$ and $\mathbf{Q}_{P}$ as follows:

$$
\begin{aligned}
\mathbf{Q}_{h}= & \left\{\mathbf{q}_{h} \in\left(V_{h}\right)^{2 \times 2}, \mathbf{q}_{h}\left(P_{k}\right)=\mathbf{q}_{h}^{T}\left(P_{k}\right), k=1, \ldots, N_{0 h}\right\} \\
\mathbf{Q}_{P h}= & \left\{\mathbf{q} \in \mathbf{Q}_{h}, q_{h 11}\left(P_{k}\right)+q_{h 22}\left(P_{k}\right) \leq 0, \alpha\left(q_{h 11}\left(P_{k}\right)+q_{h 22}\left(P_{k}\right)\right)^{2}+\right. \\
& (\alpha-1)^{2}\left(q_{h 11}\left(P_{k}\right) q_{h 22}\left(P_{k}\right)-q_{h 12}\left(P_{k}\right)^{2}\right)=0 \\
& \left.k=1, \ldots, N_{0 h}\right\}
\end{aligned}
$$


We associate on $V_{h}$ (or $V_{0 h}$ and $V_{g h}$ ) and $\mathbf{Q}_{h}$, the discrete scalar products: $(v, w)_{0 h}=\frac{1}{4} \sum_{k=1}^{N_{h}} A_{k} v\left(P_{k}\right) w\left(P_{k}\right)$ (with corresponding norm $\|v\|_{0 h}=\sqrt{(v, v)_{h}}$ ), for all $v, w \in V_{0 h}$, and $((\mathbf{S}, \mathbf{T}))_{0 h}=\frac{1}{4} \sum_{k=1}^{N_{h}} A_{k} \mathbf{S}\left(P_{k}\right): \mathbf{T}\left(P_{k}\right)$ (with corresponding norm $\left.\||\mathbf{S}|\|_{0 h}=\sqrt{((\mathbf{S}, \mathbf{S}))_{0 h}}\right)$ for all $\mathbf{S}, \mathbf{T} \in \mathbf{Q}_{h}$, where $A_{k}$ is the area of the polygonal domain which is the union of those triangles of $\mathcal{T}_{h}$ with $P_{k}$ as a common vertex.

The solution of the discrete Pucci's equation is then addressed with a nonlinear least-squares method, namely find $\left(\psi_{h}, \mathbf{p}_{h}\right) \in V_{g h} \times \mathbf{Q}_{P h}$ such that

where:

$$
J_{h}\left(\psi_{h}, \mathbf{p}_{h}\right) \leq J_{h}\left(\varphi_{h}, \mathbf{q}_{h}\right), \quad \forall\left(\varphi_{h}, \mathbf{q}_{h}\right) \in V_{g h} \times \mathbf{Q}_{P h}
$$

$$
J_{h}\left(\varphi_{h}, \mathbf{q}_{h}\right)=\frac{1}{2}\left\|\left|\mathbf{D}_{h}^{2} \varphi_{h}-\mathbf{q}_{h}\right|\right\|_{0 h}^{2}
$$

6.4. Discrete Relaxation Algorithm. The discrete relaxation algorithm reads as follows: First start with the solution of the discrete elliptic problem:

$$
-\Delta \psi_{h}^{0}=0, \quad \text { in } \Omega, \quad \psi_{h}^{0}=g, \quad \text { on } \partial \Omega .
$$

Then, for $n \geq 0$, assuming that $\psi_{h}^{n}$ is known, compute as follows $\mathbf{p}_{h}^{n}, \psi_{h}^{n+1 / 2}$ and $\psi_{h}^{n+1}$ :

with $1 \leq \omega \leq \omega_{\max }<2$.

$$
\begin{aligned}
\mathbf{p}_{h}^{n} & =\arg \min _{\mathbf{q}_{h} \in \mathbf{Q}_{P h}} J_{h}\left(\psi_{h}^{n}, \mathbf{q}_{h}\right) ; \\
\psi_{h}^{n+1 / 2} & =\arg \min _{\varphi_{h} \in V_{g h}} J_{h}\left(\varphi_{h}, \mathbf{p}_{h}^{n}\right) ; \\
\psi_{h}^{n+1} & =\psi_{h}^{n}+\omega\left(\psi_{h}^{n+1 / 2}-\psi_{h}^{n}\right),
\end{aligned}
$$

6.5. Finite Element Approximation of Local Nonlinear Problems. The finite dimensional minimization problems, discrete equivalents of (8), are approximated, at each grid point $P_{k} \in \Sigma_{h}$, by:

$$
\mathbf{p}_{h}^{n}\left(P_{k}\right)=\arg \min _{\mathbf{q} \in \mathbf{E}_{P}}\left[\frac{1}{2}|\mathbf{q}|^{2}-\mathbf{D}_{h}^{2} \psi^{n}\left(P_{k}\right): \mathbf{q} d \mathbf{x}\right] .
$$

The same Newton method presented in Section 4 applies.

6.6. Finite Element Approximation of Linear Variational Problems. The variational problems arising in the discrete version of the relaxation algorithm can be solved similarly as in the continuous case with a conjugate gradient algorithm. While keeping in mind that algorithm (13)-(15) can be transposed on the discrete level, let us focus here only the particularities that arise in the discrete case. The discrete version of (12) reads as follows: find $\psi_{h}^{n+1 / 2} \in V_{g h}$ satisfying:

$$
\left(\left(\mathbf{D}_{h}^{2} \psi_{h}^{n+1 / 2}, \mathbf{D}_{h}^{2} \varphi_{h}\right)\right)_{0 h}=\left(\left(\mathbf{p}_{h}^{n}, \mathbf{D}_{h}^{2} \varphi_{h}\right)\right)_{0 h}, \quad \forall \varphi_{h} \in V_{0 h} .
$$

The linear problem (18) may lead to excessive computer resource requirements. (indeed, to derive the linear system equivalent to (18), we need to compute-via the solution of (17)-the matrix-valued functions $\mathbf{D}_{h}^{2} w^{j}$, where the functions $w^{j}$ form a basis of $\left.V_{0 h}\right)$. To avoid this difficulty, we are going to employ an adjoint equation approach (see, e.g., [22]) to derive an equivalent formulation of (18), well-suited to a solution by a conjugate gradient algorithm, which reads 
Find $\psi_{h}^{n+1 / 2} \in V_{g h}$ such that $\left\langle\frac{\partial J_{h}}{\partial \varphi}\left(\psi_{h}^{n+1 / 2}, \mathbf{p}_{h}^{n}\right), \theta_{h}\right\rangle=0, \quad \forall \theta_{h} \in V_{0 h}$,

where $\left\langle\frac{\partial J_{h}}{\partial \varphi}(\varphi, \mathbf{q}), \theta\right\rangle$ denotes the action of the partial derivative $\frac{\partial J_{h}}{\partial \varphi}(\varphi, \mathbf{q})$ on the test function $\theta$. In order to solve (19), we first determine $D_{h i j}^{2} \varphi$ via (17). Then, we find $\lambda_{h i j} \in V_{0 h}, 1 \leq i, j \leq 2$ by solving the (adjoint) systems:

$\left(\lambda_{h i j}, \theta_{h}\right)_{0 h}+C \sum_{K \in \mathcal{T}_{h}}|K| \int_{K} \nabla \lambda_{h i j} \cdot \nabla \theta_{h} d \mathbf{x}=\left(\mathbf{p}_{h i j}-D_{h i j}^{2} \varphi, \theta_{h}\right)_{0 h}, \quad \forall \theta_{h} \in V_{0 h}$, and we can show (see, e.g., [22]) that, for all $\left(\varphi_{h}, \mathbf{p}_{h}\right) \in V_{g h} \times \mathbf{Q}_{h}$ :

$$
\left\langle\frac{\partial J_{h}}{\partial \varphi}\left(\varphi_{h}, \mathbf{p}_{h}\right), \theta_{h}\right\rangle=\int_{\Omega}\left[\sum_{i=1}^{2} \sum_{j=1}^{2} \frac{\partial \lambda_{h i j}}{\partial x_{i}} \frac{\partial \theta_{h}}{\partial x_{j}}\right] d \mathbf{x}, \quad \forall \theta_{h} \in V_{0 h}
$$

This last relationship can be used directly in the conjugate gradient algorithm (13)-(15), to solve (13) and (14). For instance, the discrete equivalent of (14) consists in finding $\bar{g}_{h}^{k} \in V_{0 h}$ such that

$$
\left(\Delta \bar{g}_{h}^{k}, \Delta \theta_{h}\right)_{0 h}=\int_{\Omega}\left[\sum_{i=1}^{2} \sum_{j=1}^{2} \frac{\partial \lambda_{h i j}}{\partial x_{i}} \frac{\partial \theta_{h}}{\partial x_{j}}\right] d \mathbf{x}, \quad \forall \theta_{h} \in V_{0 h} .
$$

\section{Numerical Experiments}

Numerical simulations are performed in the sequel to validate the proposed method, reproduce some results from [8], and expand those results to various domains and less regular examples.

7.1. Smooth Validation Example. We first consider the unit square $\Omega=$ $(0,1)^{2}$, with boundary $\partial \Omega$, and set $\mathbf{x}:=\left(x_{1}, x_{2}\right)$. The data to enforce Dirichlet boundary conditions on $\partial \Omega$ are given by:

$$
g(\mathbf{x})=-\rho(\mathbf{x})^{1-\alpha}, \quad \mathbf{x} \in \partial \Omega
$$

where $\rho(\mathbf{x})=\rho\left(x_{1}, x_{2}\right)=\left(\left(x_{1}+1\right)^{2}+\left(x_{2}+1\right)^{2}\right)^{1 / 2}$. The exact solution to $(1)$ associated with the boundary conditions (20) is thus given by

$$
\psi(\mathbf{x})=-\rho(\mathbf{x})^{1-\alpha}, \quad \mathbf{x} \in \Omega,
$$

for all values of $\alpha>1$; this solution satisfies $\psi \in C^{\infty}(\bar{\Omega}) \subset H^{2}(\Omega)$, since the singularity of (21) is located outside the domain $\Omega$.

The outer loop iteration stops after 100 iterations, or if the tolerance between successive iterates $\mathbf{u}^{k}$ and $\mathbf{u}^{k+1}$ satisfies $\left\|\mathbf{u}^{k}-\mathbf{u}^{k+1}\right\|_{0 h}<\varepsilon=10^{-7}$. When computing $\mathbf{p}_{h}^{n}$, we take $\varepsilon_{N}=10^{-5}$ as the tolerance for the Newton's method solving the discrete analogues of problem (11), and limit to $N_{i t}=100$ the number of Newton's iterations (a number that is never reached). The stopping criterion for the conjugate gradient algorithm (13)-(15) to compute $\psi_{h}^{n+1}$ is applied with $\varepsilon=10^{-5}$, with a maximal number of 100 iterations. We typically take no relaxation $(\omega=1)$. The value of the parameter $C$ is set between 1.0 and 100.0, and we consider values of $\alpha=2.0,2.5$ and 3.0 in the numerical 
results. We consider both structured and isotropic triangulations of $\Omega$ (structured meshes are obtained by splitting regular squares along the first diagonal direction, while isotropic meshes are obtained via advancing front procedures).

Figure 1 shows snapshots of the solution after 100 iterations for two isotropic meshes $(h \simeq 0.0509$ and $h \simeq 0.0093)$, and $\alpha=3.0$.
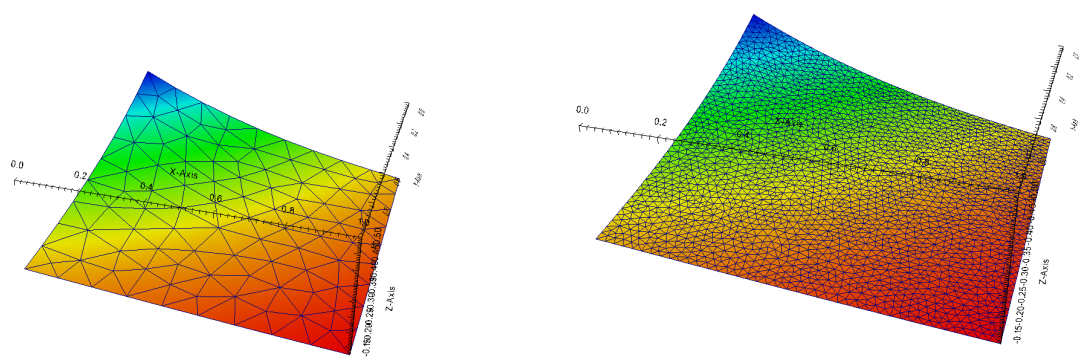

Figure 1. Smooth validation example on the unit square. Numerical approximation $\psi_{h}$ of the solution of the Pucci's equation with Dirichlet boundary data (20) and $\alpha=3.0$. Snapshots for $h \simeq 0.0509$ (left), and $h \simeq 0.0093$ (right).

In order to estimate the robustness of the algorithm with respect to curved boundaries, we also consider the same Dirichlet problem on the unit disk $\Omega=\left\{\left(x_{1}, x_{2}\right) \in \mathbb{R}^{2}: x_{1}^{2}+x_{2}^{2}<1\right\}$. Figure 2 shows snapshots of the solution after 100 iterations for two (isotropic) meshes $(h \simeq 0.0251$ and $h \simeq 0.0099$ ), and for both $\alpha=2.0$ and $\alpha=3.0$. As in [8], we observe that, when $\alpha$ increases, the convergence is more difficult to obtain, as the problem becomes more fully nonlinear, making it more difficult to solve with an elliptic solver based approach. This translates into a larger residual $\left\|\mid \mathbf{D}_{h}^{2} \psi_{h}-\mathbf{p}_{h}\right\|_{0, \Omega}$ (when $\alpha$ increases) at a fixed given number of iterations.

Convergence properties are illustrated in Figure 3 for the two different triangulations of the unit square, and for the isotropic triangulation of the unit disk. It shows a consistent first order convergence order of the method, for all three values of $\alpha=2.0,2.5$ and 3.0, and for all triangulations of the unit square and unit disk. However, the method loses some accuracy when considering unstructured meshes and curved boundaries (this is also due to the polygonal approximation of $\Omega$ ), and the convergence properties of the algorithm deteriorate slightly when the value of $\alpha$ increases (typically the number of outer iterations to reach convergence increases when $\alpha$ increases).

Let us discuss shortly the influence of the smoothing parameter $C$, by considering for instance an unstructured mesh of the unit square (with $h \simeq 0.01608$ fixed) and $\alpha=2.0$. Table 1 illustrates the residual, the error, and the number of iterations as a function of the numerical parameter $C$. It justifies the choice of $C=1$ (or below) in the sequel. Moreover, note that, for instance for the unit disk, the value of $C$ has to decrease with $h$ in some cases (typically from $C=100$ to $C=0.01$ ) to obtain the convergence behavior exhibited in Figure 3.

7.2. Regularization of Boundary Data. With the aim of reproducing results from [8], we consider $\Omega=(0,1)^{2}$ equipped with an unstructured mesh, 

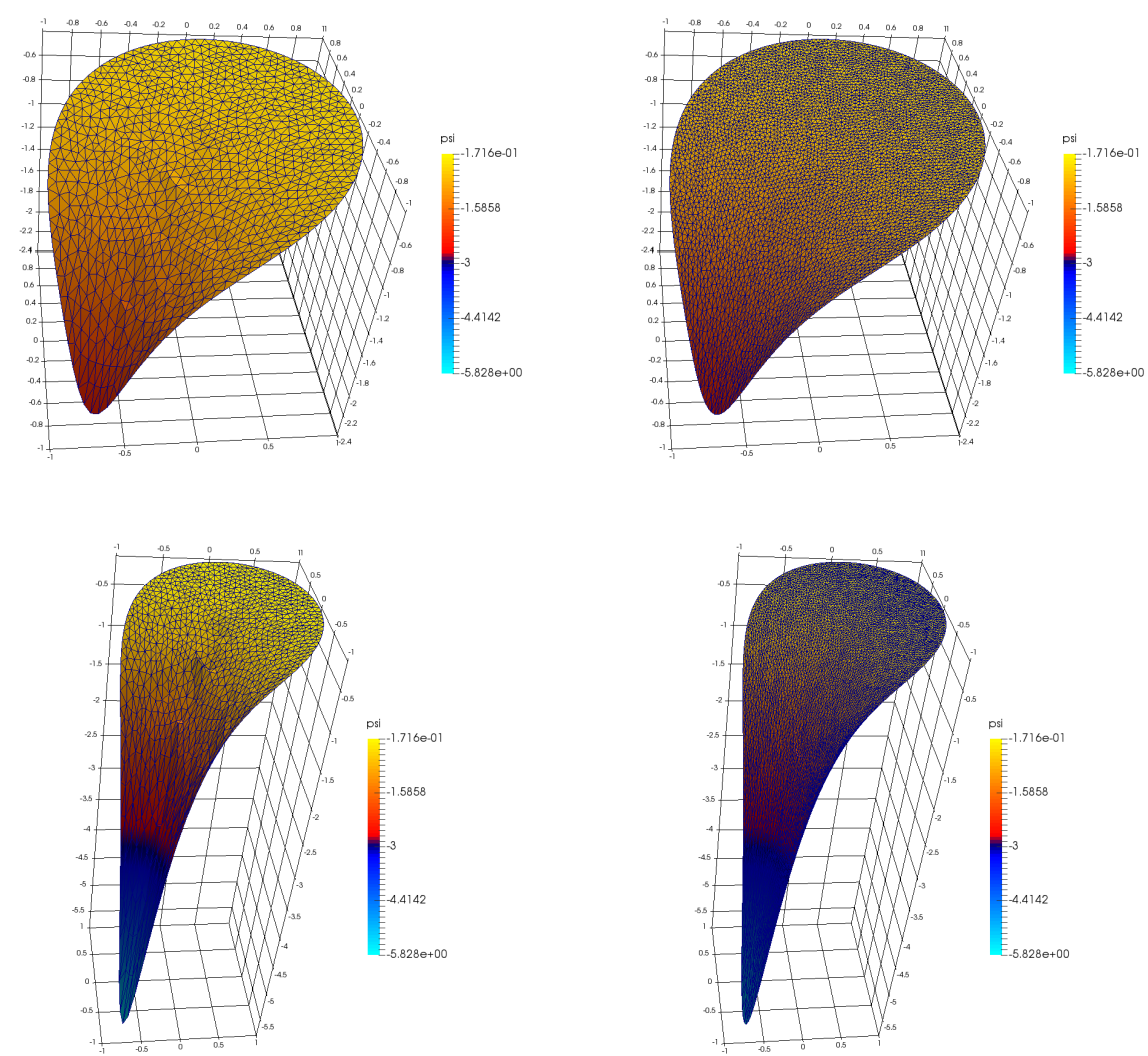

Figure 2. Smooth validation example on the unit disk. Numerical approximation $\psi_{h}$ of the solution of the Pucci's equation with Dirichlet boundary data (20) and $\alpha=2.0$ (first row) and $\alpha=3.0$ (second row). Snapshots for $h \simeq 0.0251$ (left), and $h \simeq 0.0099$ (right).
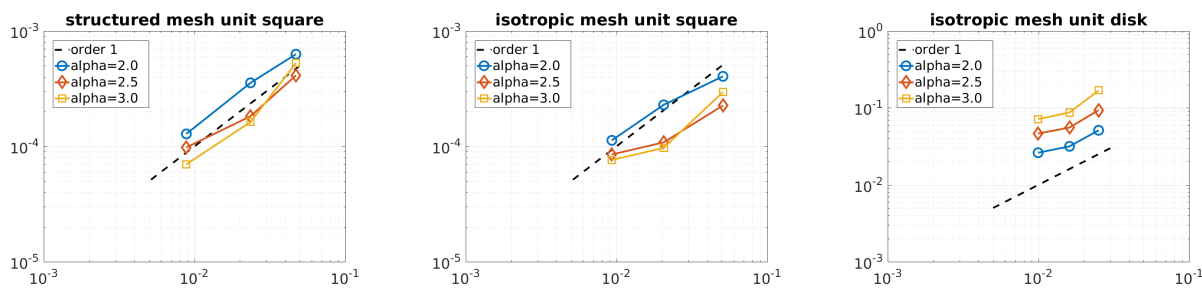

Figure 3. Smooth validation example. Convergence of the approximation error $\left\|\psi_{h}-\psi\right\|_{0, \Omega}$ for $\alpha=2.0,2.5$ and 3.0 using structured (left) and isotropic (middle) triangulations of the unit square, and isotropic triangulations of the unit disk (right).

and Dirichlet boundary data given by the indicator function of a subset of the boundary $\partial \Omega$ : 
Table 1. Smooth validation example. Sensitivity analysis of the numerical results with respect to the smoothing parameter $C$. Isotropic mesh of the unit square $(h \simeq 0.01608)$ and $\alpha=$ 2.0 .

\begin{tabular}{|c|c|c|c|}
\hline $\mathrm{C}$ & $\left\|\psi_{h}-\psi\right\|_{0, \Omega}$ & || $\mathbf{D}_{h}^{2} \psi_{h}-\mathbf{p}_{h}\left|\left\|_{0, \Omega} /\right\|\right| \mathbf{p}_{h}|\||_{0, \Omega}$ & $\#$ outer iter. \\
\hline $10^{2}$ & $0.5045 \cdot 10^{-1}$ & $0.6641 \cdot 10^{-2}$ & 50 \\
$10^{1}$ & $0.3497 \cdot 10^{-1}$ & $0.6747 \cdot 10^{-2}$ & 10 \\
$10^{0}$ & $0.3167 \cdot 10^{-1}$ & $0.6705 \cdot 10^{-2}$ & 7 \\
$10^{-1}$ & $0.3086 \cdot 10^{-1}$ & $0.6705 \cdot 10^{-2}$ & 6 \\
$10^{-2}$ & $0.2967 \cdot 10^{-1}$ & $0.6511 \cdot 10^{-2}$ & 9 \\
$10^{-3}$ & $0.2964 \cdot 10^{-1}$ & $0.6528 \cdot 10^{-2}$ & 6 \\
$10^{-4}$ & $0.2973 \cdot 10^{-1}$ & $0.6527 \cdot 10^{-2}$ & 6 \\
\hline
\end{tabular}

$$
g(\mathbf{x})= \begin{cases}0 & \text { if } \mathbf{x} \in \bigcup_{i=1}^{4} \Gamma_{i} \\ 1 & \text { if } \mathbf{x} \in \partial \Omega \backslash \bigcup_{i=1}^{4} \Gamma_{i}\end{cases}
$$

where

$$
\begin{aligned}
& \Gamma_{1}=\left\{\mathbf{x}=\left(x_{1}, x_{2}\right) \in \partial \Omega: 1 / 4<x_{1}<3 / 4, x_{2}=0\right\} ; \\
& \Gamma_{2}=\left\{\mathbf{x}=\left(x_{1}, x_{2}\right) \in \partial \Omega: x_{1}=1,1 / 4<x_{2}<3 / 4\right\} ; \\
& \Gamma_{3}=\left\{\mathbf{x}=\left(x_{1}, x_{2}\right) \in \partial \Omega: 1 / 4<x_{1}<3 / 4, x_{2}=1\right\} ; \\
& \Gamma_{4}=\left\{\mathbf{x}=\left(x_{1}, x_{2}\right) \in \partial \Omega: x_{1}=0,1 / 4<x_{2}<3 / 4\right\} .
\end{aligned}
$$

The boundary data $g$ is a (multi-)step function, and thus $g \notin H^{3 / 2}(\Omega)$. Therefore the corresponding Pucci's equation (1) has no solution in $H^{2}(\Omega)$. In order to address a problem with a solution, we consider a mollified version, $g_{\delta} \in H^{3 / 2}(\Omega)$, of the boundary data. It is regularized as follows, for instance on the edge $\left\{\mathbf{x}=\left(x_{1}, x_{2}\right) \in \partial \Omega: 0<x_{1}<0, x_{2}=0\right\}$ :

$$
g_{\delta}(\mathbf{x})= \begin{cases}1 & \text { if } x_{1}<1 / 4-\delta \text { or } 3 / 4+\delta<x_{1} \\ 0 & \text { if } 1 / 4+\delta<x_{1}<3 / 4-\delta ; \\ \frac{1}{2}\left(1-\sin \left(\frac{\pi}{2} \frac{x_{1}-1 / 4}{\delta}\right)\right) & \text { if } 1 / 4-\delta<x_{1}<1 / 4+\delta ; \\ \frac{1}{2}\left(1+\sin \left(\frac{\pi}{2} \frac{x_{1}-3 / 4}{\delta}\right)\right) & \text { if } 3 / 4-\delta<x_{1}<3 / 4+\delta ;\end{cases}
$$

(with a similar definition on the other edges of $\partial \Omega$ ). In the sequel, we consider $\delta=1 / 16$. Figure 4 illustrates the numerical approximation $\psi_{h}$ for various values of $\alpha$, while Figure 5 illustrates cuts of the graph of the solution along $x_{1}=0.5$ and $x_{1}=x_{2}$ respectively. These results compare well with [8], and strongly suggest convergence to a limit solution. Together with Figure 6, they show that $\psi_{h}:=\psi_{h}(\alpha)$ is an increasing function of $\alpha$.

Table 2 illustrates the number of iterations of the relaxation algorithm for various meshes and values of the parameter $\alpha$. It shows that the number of iterations increases with $\alpha$ (since the fully nonlinear problem becomes more difficult to solve with linear elliptic solvers), but that the method remains 

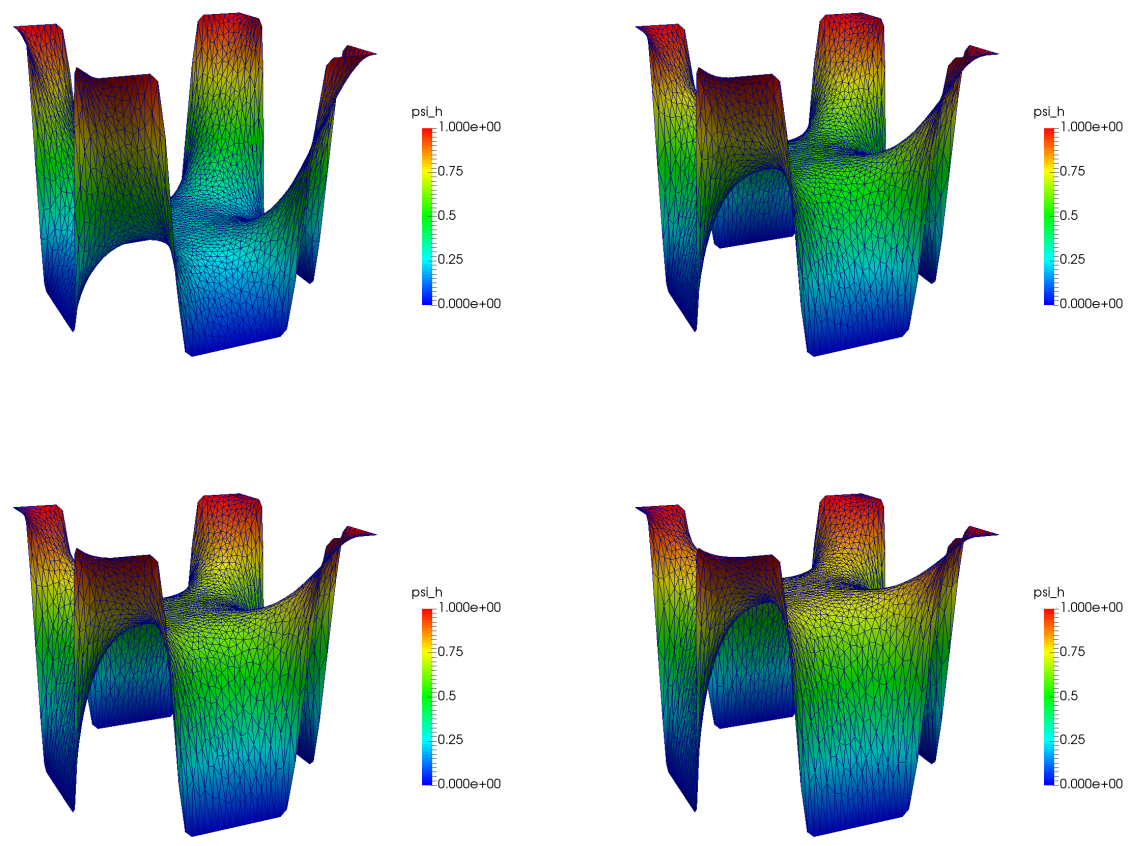

Figure 4. Regularization of the boundary data. Snapshot of the numerical approximation for $\alpha=1.005$ (top left), $\alpha=2.0$ (top right), $\alpha=2.5$ (bottom left), and $\alpha=3.0$ (bottom right), for an isotropic triangulation of the unit square $(h \simeq 0.009327)$
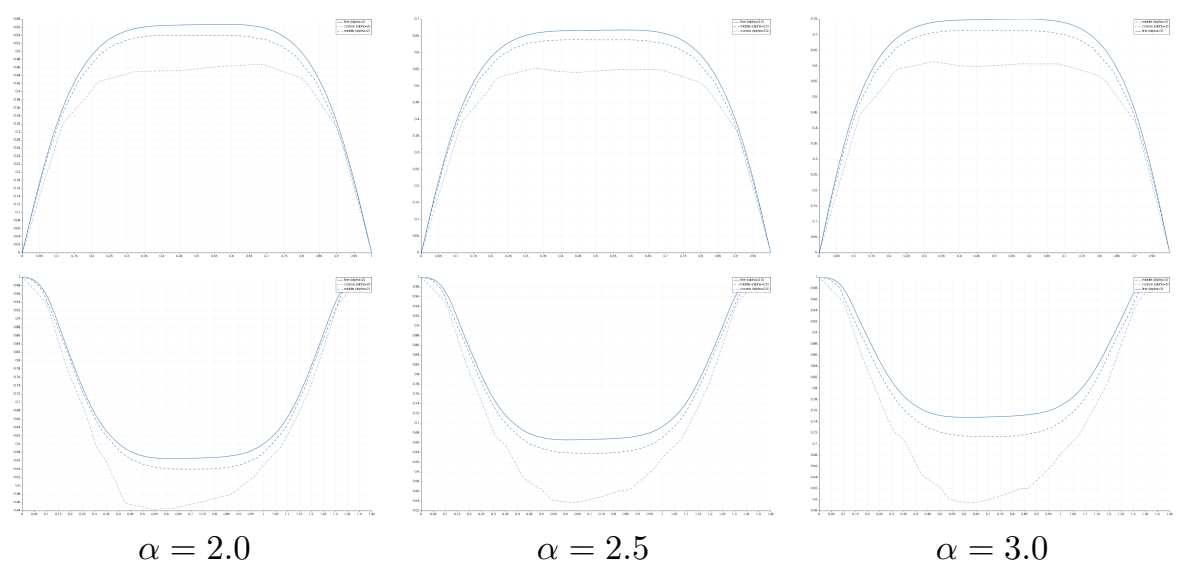

Figure 5. Regularization of the boundary data. Cuts of the graph of the numerical approximation along $x_{1}=0.5$ (first row) and $x_{1}=x_{2}$ (second row) for an isotropic triangulation of the unit square with mesh size $h \simeq 0.0509$ (dash dotted line), $h \simeq 0.0205$ (dashed line) and $h \simeq 0.0093$ (plain line). $\alpha=2.0$ (left), $\alpha=2.5$ (middle), and $\alpha=3.0$ (right). 


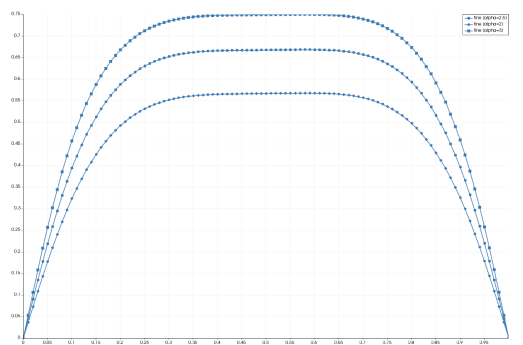

$x_{1}=0.5$

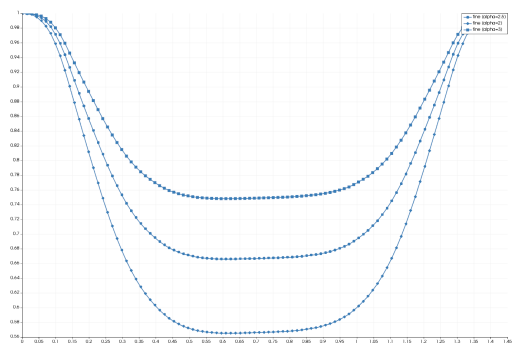

$x_{1}=x_{2}$

Figure 6. Regularization of the boundary data. Cuts of the graph of the numerical approximation along $x_{1}=0.5$ (left) and $x_{1}=x_{2}$ (right) for an isotropic triangulation of the unit square $(h \simeq 0.0093)$, and $\alpha=2.0$ (circles), 2.5 (diamonds), and 3.0 (squares).

stable as the number of iterations remains roughly constant when the mesh size decreases.

Table 2. Regularization of the boundary data. Number of outer iterations of the least-squares algorithm as a function of mesh size and $\alpha$.

\begin{tabular}{|c|c|c|c|}
\hline & $\alpha=2$ & $\alpha=2.5$ & $\alpha=3$ \\
\hline$h \simeq 0.0509$ & 20 & 28 & 35 \\
$h \simeq 0.0205$ & 18 & 26 & 35 \\
$h \simeq 0.0093$ & 21 & 28 & 38 \\
\hline
\end{tabular}

7.3. Homogenization Example with Variable Periodic Coefficient. We consider $\Omega=(0,1)^{2}$, equipped with a isotropic triangular mesh, and extend our methodology to a variable, periodic, coefficient $\alpha$, like the coefficients that may appear in homogenization theory. In order to do do, let us consider again a boundary data $g$ representing the characteristic function of a subset of the boundary $\partial \Omega$ :

$$
g(\mathbf{x})= \begin{cases}0 & \text { if } \mathbf{x} \notin \Gamma_{1} \\ 1 & \text { if } \mathbf{x} \in \Gamma_{1}\end{cases}
$$

where $\Gamma_{1}=\left\{\left(x_{1}, x_{2}\right) \in \mathbb{R}^{2}, x_{1}=1,1 / 4<x_{2}<3 / 4\right\}$. We consider a variable, periodic, coefficient $\alpha$ defined by:

$$
\alpha\left(x_{1}, x_{2}\right)=\frac{1}{2}\left(\alpha_{1}+\alpha_{2}\right)+\frac{1}{2}\left(\alpha_{1}-\alpha_{2}\right) \cos \left(2 m \pi x_{1}\right) \cos \left(2 n \pi x_{2}\right),
$$

where $\alpha_{1}, \alpha_{2}>1.0$ are two given values, and $m, n \in \mathbb{N}$. The coefficient $\alpha$ thus oscillates periodically between the two values $\alpha_{1}$ and $\alpha_{2}$, as illustrated in Figure 7 when $\alpha_{1}=2.0, \alpha_{2}=3.0$ and $m=n=8$.

Again, the boundary data $g$ is mollified, as follows, to provide a boundary data in $H^{s}(\partial \Omega), s<3 / 2$, and Lipschitz continuous: 


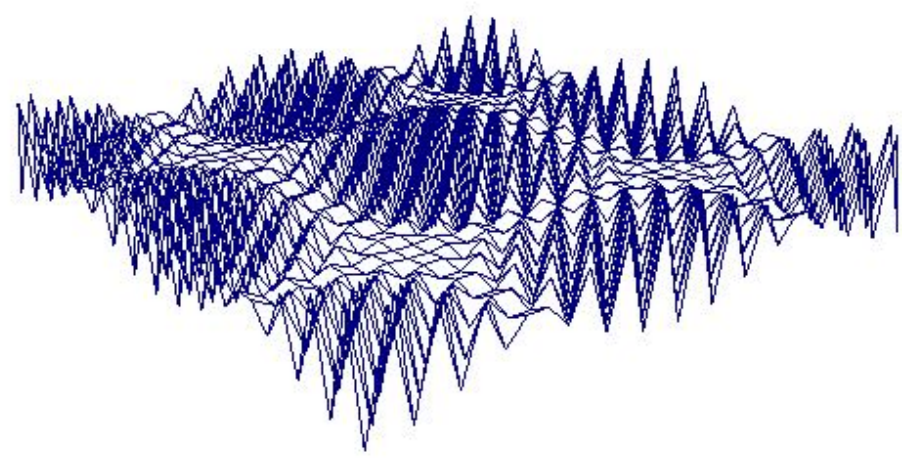

Figure 7. Homogenization example with variable periodic coefficient $\alpha$. Visualization of $\alpha$ when $\alpha_{1}=2.0, \alpha_{2}=3.0$ and $m=n=8$.

$$
g_{\delta}(\mathbf{x})= \begin{cases}1 & \text { if } \mathbf{x} \in \Gamma ; \\ \left(x_{2}-1 / 4+\delta\right) / \delta & \text { if } x_{1}=1, \quad 1 / 4-\delta<x_{2}<1 / 4 \\ \left(3 / 4+\delta-x_{2}\right) / \delta & \text { if } x_{1}=1, \quad 3 / 4<x_{2}<3 / 4+\delta \\ 0 & \text { if otherwise }\end{cases}
$$

Figure 8 illustrates the numerical approximation $\psi_{h}$ for various values of $m$ and $n\left(m, n=8,16, \alpha_{1}=2, \alpha_{2}=3\right)$ for an isotropic discretization of the unit square $(h \simeq 0.0093)$. Figure 9 illustrates cuts of the graph of the solutions along $x_{1}=0.5$ and $x_{1}=x_{2}$ respectively, compared with the cut of the graph of the solution obtained for the same (regularized) boundary data and a constant coefficient $\alpha=0.5\left(\alpha_{1}+\alpha_{2}\right)=2.5$. These results strongly suggest that the solution of the homogenization problem converges to the solution of the Pucci's equation with the average value $\alpha=2.5$ when $m, n \rightarrow \infty$.

7.4. Non-smooth Solution on a Non-Convex Domain. In order to assess the least-squares approach for a non-convex domain, let us consider the domain obtained when removing a piece of angular size $\theta$ from the unit disk domain, namely:

$$
\Omega=\left\{\left(x_{1}, x_{2}\right): \sqrt{x_{1}^{2}+x_{2}^{2}}<1\right\} \backslash\left\{\left(x_{1}, x_{2}\right): x_{1} \geq 0,\left|\frac{x_{2}}{x_{1}}\right| \leq \tan (\theta)\right\} .
$$

Let us also define $\omega:=2 \pi-2 \theta$, and equip $\Omega$ with an isotropic discretization. The Dirichlet boundary conditions on $\partial \Omega$ are given by:

$$
g(\mathbf{x})=-\rho(\mathbf{x})^{1-\alpha}, \quad \mathbf{x} \in \partial \Omega,
$$

where $\rho(\mathbf{x})=\rho\left(x_{1}, x_{2}\right)=\left(\left(x_{1}-\xi\right)^{2}+x_{2}^{2}\right)^{1 / 2}$, and $\xi>0$ is a given parameter, enforcing the singularity to be outside the computational domain. The exact solution to (1) associated with those boundary conditions is $\psi(\mathbf{x})=-\rho(\mathbf{x})^{1-\alpha}$, for all $\mathbf{x} \in \Omega$, and for all values of $\alpha>1$. This solution satisfies $\psi \in C^{\infty}(\bar{\Omega}) \subset$ $H^{2}(\Omega)$. 


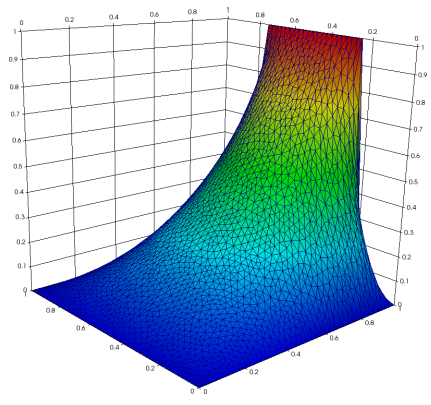

$(m, n)=(8,8)$

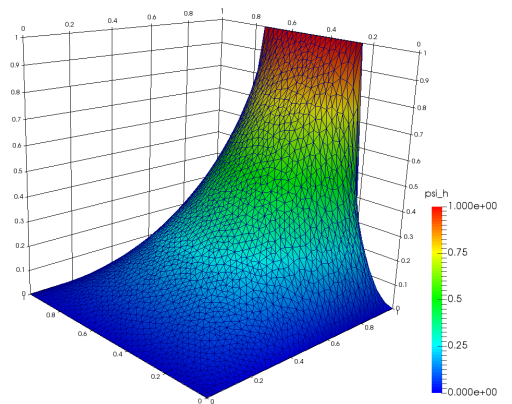

$(m, n)=(16,8)$

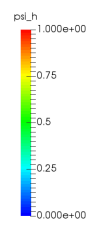

Figure 8. Homogenization example with variable periodic coefficient $\alpha$. Snapshot of the graph of the numerical approximation for $(m, n)=(8,8)$ (top left), $(m, n)=(8,16)$ (top right), $(m, n)=(16,8)$ (bottom left), and $(m, n)=(16,16)$ (bottom right)

We consider here $\theta=\pi / 100$, and an isotropic discretization of the domain with $h \simeq 0.0191$, together with $\alpha=2.0$, and we vary the parameter $\xi$. Figure 10 illustrates isovalues of the approximated solution to the Pucci's equation for $\xi=1.0,0.5,0.01$ and 0.0001 . Note that, when $\xi \rightarrow 0$, the singularity gets closer to the computational domain; thus the minimal value of the function tends to $-\infty$, and the convergence of the algorithm is expected to be more difficult to obtain. One can observe that the location of the singularity is indeed shifting, but remains very localized in space. Table 3 illustrates this effect, but shows that the increase in the number of outer iterations remains reasonable when $\xi \rightarrow 0$. Not surprisingly, the computed error (in the $L^{2}$-norm) is impacted, although more than $90 \%$ of the error is concentrated in a patch of elements (triangles) adjacent to the vertex in the re-entrant corner.

\section{Conclusions and Perspectives}

A least-squares approach for the solution of a Pucci's equation in two dimensions of space has been presented. This versatile method relies on a decoupling 


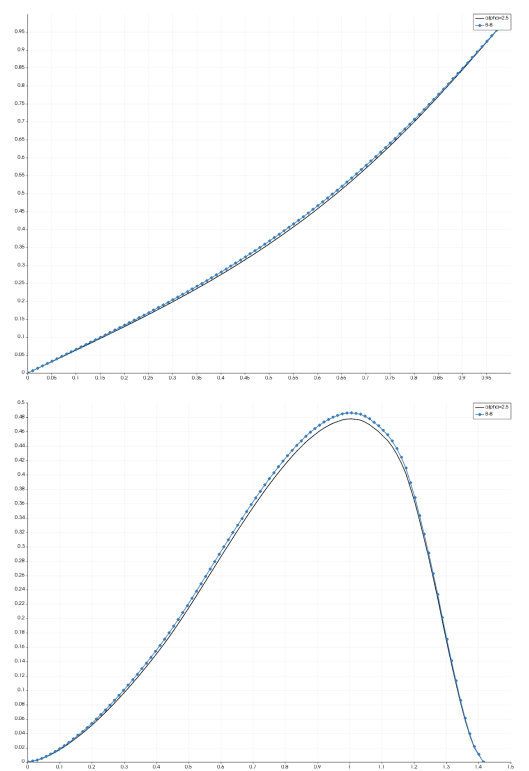

$(8,8)$
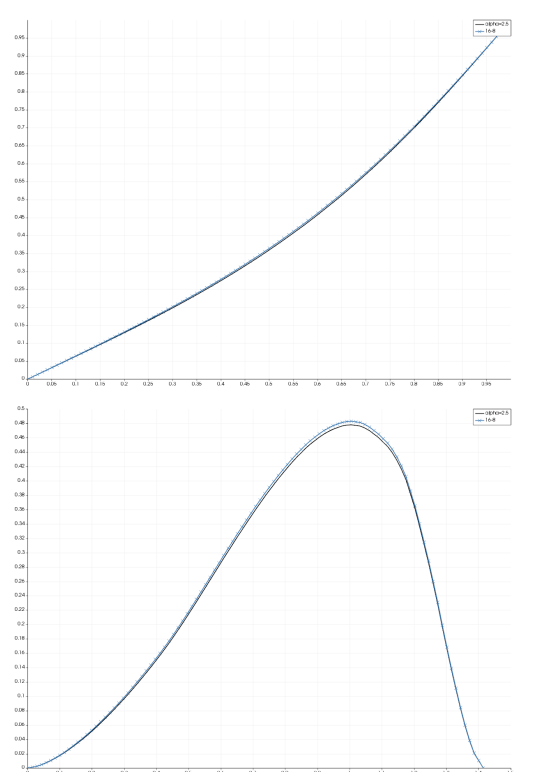

$(16,8)$

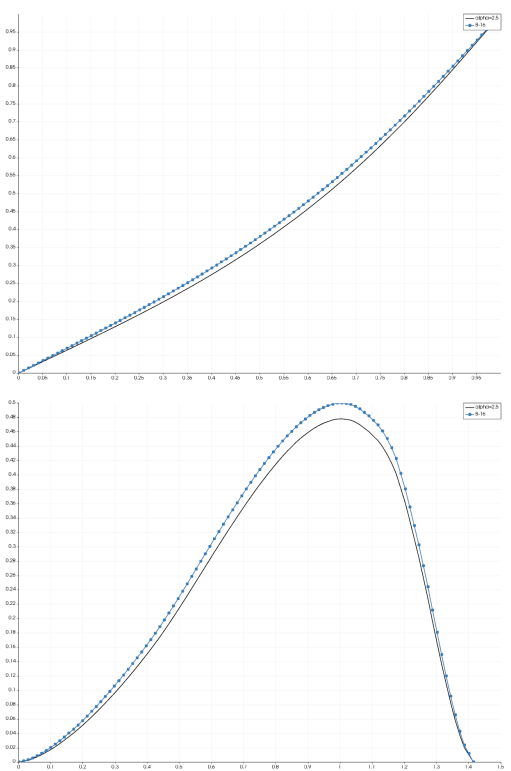

$(8,16)$

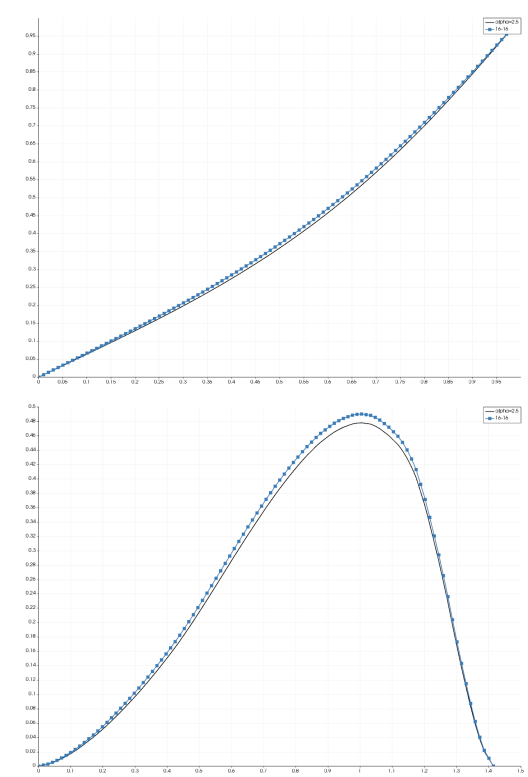

$(16,16)$

Figure 9. Homogenization example with variable periodic coefficient $\alpha$. Cuts of the graph of the numerical approximation for $x_{2}=0.5$ (top), and $x_{1}=x_{2}$ (bottom). Top left: $(m, n)=(8,8)$ (diamonds), top right: $(m, n)=(8,16)$ (circles), bottom left: $(m, n)=(16,8)$ (crosses), and bottom right: $(m, n)=(16,16)$ (squares). On all graphs, the black plain curve corresponds to the limit solution with $\alpha=2.5$. 
Table 3. Non-smooth solution on a non-convex domain. Influence of the location of the singularity (parametrized by $\xi$ ) on the performance of the numerical algorithm.

\begin{tabular}{|c|c|c|c|c|}
\hline$\xi$ & 1 & 0.5 & $10^{-2}$ & $10^{-4}$ \\
\hline $\min \psi_{h}$ & -31.84 & -63.69 & -100 & -1000 \\
\hline \# iterations & 24 & 26 & 35 & 39 \\
\hline$\left\|\psi_{h}-\psi\right\|_{0, \Omega}$ & $0.6097 \cdot 10^{-1}$ & $0.6760 \cdot 10^{0}$ & $0.3555 \cdot 10^{1}$ & $0.4829 \cdot 10^{3}$ \\
\hline
\end{tabular}
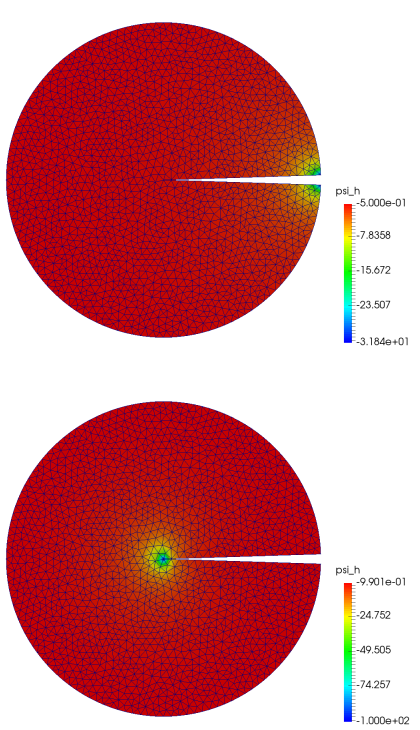
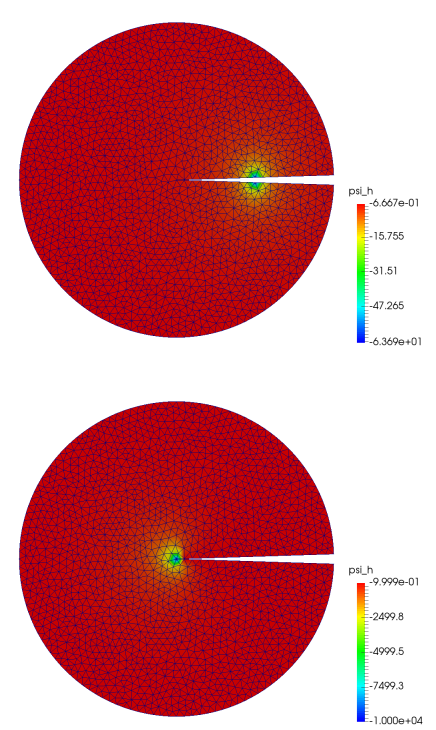

Figure 10. Non-smooth solution on a non-convex domain. Contour maps of the numerical approximation $\psi_{h}$ of the solution to the Pucci's equation, for $\xi=1.0$ (top left), $\xi=0.5$ (top right), $\xi=0.01$ (bottom left), and $\xi=0.0001$ (bottom right), for an isotropic mesh of typical size $h \simeq 0.0191$.

of the variational aspects of the problem from the local nonlinearities, and allows to expand existing results from the literature to arbitrary meshes and arbitrary domains. Mixed finite elements have been used for the space discretization. Numerical results have shown the flexibility and the robustness of the proposed approach. In particular, non-smooth problems in domains that are non-convex or with curved boundaries have been treated.

Perspectives may include the derivation of a similar approach for the solution of the Pucci's equation in three space dimensions, namely looking for a function $\psi: \Omega \rightarrow \mathbb{R}$ satisfying

$$
\alpha \sum \lambda^{+}+\sum \lambda^{-}=0 \text { in } \Omega \subset \mathbb{R}^{3},
$$

with Dirichlet boundary conditions, and where $\lambda^{+}$and $\lambda^{-}$are the positive and negative eigenvalues of the Hessian $\mathbf{D}^{2} \psi$ respectively. The difficulty consists in writing this equation as a function of the components of the Hessian the same way (2) was derived in the $2 \mathrm{D}$ case. Once this step is done, the iterative algorithm is similar, and discrepancies appear for the local problem, while the 
linear variational problems are unchanged (the same way [5] is an extension of [6] in three space dimensions).

\section{Acknowledgments}

This work has been supported by the Swiss National Science Foundation (Grant SNF 165785), and the National Science Foundation (Grant NSF DMS0913982). The author thanks Prof. Roland Glowinski (University of Houston and Hong Kong Baptist University) and M. Samuel Dubuis (EPFL) for helpful comments and discussions.

\section{References}

[1] A. D. Aleksandrov. Uniqueness conditions and estimates for the solution of the Dirichlet problem. Amer. Math. Soc. Trans., 68:89-119, 1968.

[2] J. D. Benamou, B. D. Froese, and A. M. Oberman. Numerical solution of the optimal transportation problem using the Monge-Ampère equation. J. Comp. Phys., 49(4):107-126, 2014.

[3] F. Brezzi and M. Fortin. Mixed and Hybrid Finite Element Methods. Series in Computational Mathematics. Springer-Verlag, New York, 1991.

[4] J. Busca, M. Esteban, and A. Quaas. Nonlinear eigenvalues and bifurcation problems for Pucci's operator. Ann. Inst. H. Poincar Anal. Non Lineaire, 2(22):187-206, 2005.

[5] A. Caboussat, R. Glowinski, and D. Gourzoulidis. A leastsquares/relaxation method for the numerical solution of the threedimensional elliptic Monge-Ampère equation. J. Sci. Comp., 77(1):53-78, 2018.

[6] A. Caboussat, R. Glowinski, and D. C. Sorensen. A least-squares method for the numerical solution of the Dirichlet problem for the elliptic MongeAmpère equation in dimension two. ESAIM: Control, Optimization and Calculus of Variations, 19(3):780-310, 2013.

[7] L. A. Caffarelli and X. Cabré. Fully Nonlinear Elliptic Equations. American Mathematical Society, 1995.

[8] L. A. Caffarelli and R. Glowinski. Numerical solution of the Dirichlet problem for a Pucci equation in dimension two. Application to homogenization. J. Numer. Math., 16(3):185-216, 2008.

[9] E. J. Dean and R. Glowinski. Numerical solution of a two-dimensional elliptic Pucci's equation with Dirichlet boundary conditions: a least-squares approach. C. R. Acad. Sci. Paris, Sér. I, 341:374-380, 2005.

[10] E. J. Dean and R. Glowinski. On the numerical solution of the elliptic Monge-Ampère equation in dimension two: A least-square approach. In R. Glowinski and P. Neittaanmäki, editors, Partial Differential Equations: Modeling and Numerical Simulation, volume 16 of Computational Methods in Applied Sciences, pages 43-63. Springer, 2008.

[11] B. Engquist, B. D. Froese, and Y. Yang. Optimal transport for seismic full waveform inversion. Communications in Mathematical Science, 14(8):2309-2330, 2016.

[12] P. Felmer and A. Quaas. Some recent results on equations involving the Pucci's extremal operators. Contributions to nonlinear analysis, 66:263281, 2006.

[13] P. L. Felmer, A. Quaas, and M. Tang. On uniqueness for nonlinear elliptic equation involving the Pucci's extremal operator. J. Diff. Eq., 226:80-98, 
2006.

[14] X. Feng and M. Neilan. A modified characteristic finite element method for a fully nonlinear formulation of the semigeostrophic flow equations. SIAM J. Numer. Anal., 47(4):2952-2981, 2009.

[15] X. Feng, M. Neilan, and R. Glowinski. Recent developments in numerical methods for fully nonlinear 2nd order PDEs. SIAM Review, 55:1-64, 2013.

[16] X. Feng, M. Neilan, and A. Prohl. Error analysis of finite element approximations of the inverse mean curvature flow arising from the general relativity. Numer. Math., 108:93-119, 2007.

[17] B. D. Froese and A. M. Oberman. Convergent filtered schemes for the Monge-Ampère partial differential equation. SIAM J. Numer. Anal., 51(1):423-444, 2013.

[18] R. Glowinski. Finite Element Method For Incompressible Viscous Flow, volume IX of Handbook of Numerical Analysis (P.G. Ciarlet, J.L. Lions eds), pages 3-1176. Elsevier, Amsterdam, 2003.

[19] R. Glowinski. Numerical Methods for Nonlinear Variational Problems. Springer-Verlag, New York, NY, second edition, 2008.

[20] R. Glowinski. Variational Methods for the Numerical Solution of Nonlinear Elliptic Problems. SIAM, Philadelphia, PA, 2015.

[21] R. Glowinski, S. Leung, and J. Qian. A novel method for the numerical solution of the Monge-Ampère equation, 2017. Private communication.

[22] R. Glowinski, J.-L. Lions, and J. W. He. Exact and Approximate Controllability for Distributed Parameter Systems: A Numerical Approach. Encyclopedia of Mathematics and its Applications. Cambridge University Press, 2008.

[23] R. Glowinski and P. Le Tallec. Augmented Lagrangians and OperatorSplitting Methods in Nonlinear Mechanics. SIAM, Philadelphia, 1989.

[24] C. E. Gutiérrez. The Monge-Ampère Equation. Birkhaüser, Boston, 2001.

[25] H. Ishii and P.-L. Lions. Viscosity solutions of fully nonlinear second-order elliptic partial differential equations. J. Differential Equations, 83(1):26$78,1990$.

[26] O. Lakkis and T. Pryer. A finite element method for fully nonlinear elliptic problems. SIAM J. Sci. Comput., 35(4):A2025-A2045, 2013.

[27] F. Leoni. Homogeneous solutions of extremal Pucci's equations in planar cones. J. Diff. Eq., 263:863-879, 2016.

[28] A. Oberman. Wide stencil finite difference schemes for the elliptic MongeAmpère equations and functions of the eigenvalues of the Hessian. Discrete and Continuous Dynamical Systems, B, 10(1):221-238, 2008.

[29] M. Picasso, F. Alauzet, H. Borouchaki, and P.-L. George. A numerical study of some Hessian recovery techniques on isotropic and anisotropic meshes. SIAM J. Sci. Comp., 33:1058-1076, 2011.

[30] A. Quaas and B. Sirakov. Principal eigenvalues and the Dirichlet problem for fully nonlinear elliptic operators. Adv. Math., 218(1):105-135, 2008.

[31] H. Schaeffer and T. Y. Hou. An accelerated method for nonlinear elliptic PDE. J. Sci. Comp., 69:556-580, 2016.

[32] S. Stojanovic. Optimal momentum hedging via Monge-Ampère PDEs and a new paradigm for pricing options. SIAM J. Control and Optimization, 43:1151-1173, 2004.

[33] R. A. Tapia, J. E. Dennis Jr, and J. P. Schaefermeyer. Inverse, shifted inverse, and Rayleigh quotient iteration as Newton's method. SIAM Review, to appear, 2017. 
[34] A. N. Tychonoff. The regularization of incorrectly posed problems. Doklady Akad. Nauk. SSSR, 153:42-52, 1963.

Geneva School of Business Administration (Haute Ecole de Gestion de Genève), University of Applied Sciences Western Switzerland (HES-SO), Rue de la Tambourine 17, CH-1227 Carouge, Email : alexandre.caboussat@hesge.ch 